\title{
Inflammation and autoimmunity in atherosclerosis
}

\author{
Stanislaw Surma ${ }^{1,2}$ ID , Krzysztof J. Filipiak ${ }^{3}$ ID \\ ${ }^{1}$ Faculty of Medical Sciences in Katowice, Medical University of Silesia in Katowice, Poland \\ ${ }^{2}$ Club of Young Hypertensiologists, Polish Society of Hypertension \\ ${ }^{3}$ Institute of Clinical Sciences, Maria Sklodowska-Curie Medical Academy, Warsaw, Poland
}

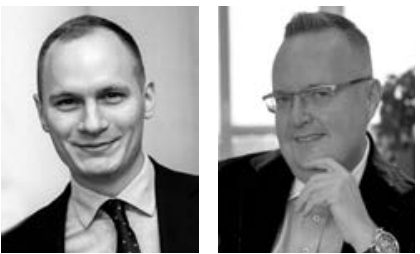

\section{Introduction}

Atherosclerosis is a process whose onset can be observed even in the foetal period. A number of risk factors, such as hyperlipidaemia, hyperhomocysteinaemia, arterial hypertension, hyperuricaemia, smoking, metabolic syndrome, hypertriglyceridaemia, and diabetes, accelerate the progression of atherosclerotic lesions leading to the development of atherosclerotic cardiovascular disease (ASCVD) [1].

Atherosclerotic cardiovascular disease is defined as coronary artery disease (CAD), cerebrovascular disease, or peripheral arterial disease of atherosclerotic origin. This disease represents the number one cause of morbidity and mortality worldwide. The number of patients with cardiovascular diseases in the world in 2019 was 523 million, while the number of deaths due to them reached 18.6 million [2].

\section{Atherosclerosis - general look on pathogenesis}

The pathogenesis of atherosclerosis is a multifactorial process involving large and medium-sized arteries, the head of the aorta, and coronary vessels, and it occurs especially in the places of their branches and bifurcations. According to modern concepts, atherosclerosis is a consequence of a long-term defence reaction of the organism, which increases with time, in the form of a chronic inflammatory response, leading to degenerative and productive changes in the inner and middle layers of the arteries. Atherosclerosis is a complex inflammatory disease involving aberrant immune and tissue-healing responses, which begins with endothelial dysfunction and ends with plaque development, instability, and rupture, which leads to MI, stroke, or critical ischaemia of the lower limbs [3].

\section{Atherosclerosis in patients with rheumatological diseases}

Many authors indicate that atherosclerosis is an auto-inflammatory disease but also an autoimmune disease, because it produces antibodies to oxidized low-density lipoprotein (oxLDL) [4, 5]. It should be noted that the progression of atherosclerosis is particularly pronounced in patients with autoimmune diseases (Table I).

Table I. The risk of atherosclerotic cardiovascular disease in people with selected autoimmune diseases

\begin{tabular}{|lcc|}
\hline Autoimmune disease - examples & $\begin{array}{c}\text { Estimated risk of ASCVD - calculated as OR/HR/RR - } \\
\text { from different papers }\end{array}$ & References \\
\hline Type 1 diabetes mellitus & $9.38(95 \% \mathrm{Cl}: 5.56-15.82)$ & {$[6]$} \\
\hline Systemic lupus erythematosus & $3.39(95 \% \mathrm{Cl}: 2.15-5.35)$ & {$[7]$} \\
\hline Rheumatoid arthritis & $2.97(95 \% \mathrm{Cl}: 1.15-7.68)$ & {$[8]$} \\
\hline Systemic sclerosis & $2.25(95 \% \mathrm{Cl}: 1.59-3.18)$ & {$[9]$} \\
\hline Psoriasis & $1.78(95 \% \mathrm{Cl}: 1.51-2.11)$ & {$[10]$} \\
\hline Hashimoto's thyroiditis & $1.44(95 \% \mathrm{Cl}: 1.05-1.99)$ & {$[11]$} \\
\hline Multiple sclerosis & $1.28(95 \% \mathrm{Cl}: 1.09-1.51)$ & {$[12]$} \\
\hline
\end{tabular}

$A S C V D$ - atherosclerotic cardiovascular disease, $H R$ - hazard ratio, $O R$ - odds ratio, $R R$ - relative risk.

Address for correspondence:

Krzysztof J. Filipiak, Institute of Clinical Sciences, Maria Sklodowska-Curie Medical Academy in Warsaw, Pałac Lubomirskich, 10 Zelaznej Bramy Sq., 00-136, Warsaw, Poland, e-mail: krzysztof.filipiak@uczelniamedyczna.com.pl

Submitted: 18.01.2022; Accepted: 24.01.2022 
Based on the data presented in Table I, it should be emphasized that autoimmune diseases are a strong independent risk factor for ASCVD. It should be stressed that the presence of autoimmune inflammatory diseases (such as rheumatoid arthritis, systemic lupus, or psoriasis) is an indication for early and regular lipid profile control and should be included in the cardiovascular risk stratification [13].

Importantly, systemic autoimmune diseases and atherosclerosis share common pathogenic pathways (chronic inflammatory background mediated by the toll-like receptors and inflammasome Nucleotide-binding oligomerization domain, Leucine rich Repeat and Pyrin domain containing 3/interleukin 1 (NLRP3/IL-1) pathways as seen in auto-inflammatory diseases and endothelial dysfunction) [5]. Moreover, autoantibodies (an essential component of autoimmune diseases) may ultimately cause structural and irreversible arterial wall damage with subsequent atherosclerotic plaque development and rupture [5]. It is worth adding that oxLDL and anti- $\beta_{2}$-glycoprotein I $\left(\beta_{2}\right.$-GPI) are both inflammatory (innate) and immunogenic (adaptive) molecules.

One possible role for these molecules is that they can serve as biological linkers to link the progression from chronic inflammation to a complete autoantibody response in the later stages of atherosclerosis. When autoantibodies are present, rheumatoid arthritis patients have an accelerated atherosclerosis compared to rheumatoid arthritis patients who do not have these antibodies. Similarly, autoantibodies in systemic lupus erythematosus exacerbate atherosclerosis [5].

In rheumatic diseases, atherosclerosis can be accelerated by both systemic inflammation and local vasculitis. Levels of pro-inflammatory cytokines such as TNF- $\alpha$, IL-6, IL-17, and IFN1 are often chronically elevated in rheumatic diseases, further exacerbating endothelial dysfunction, macrophage activation, and thrombosis [3]. A characteristic feature of rheumatological diseases is the production of autoantibodies, some of which may be deposited directly in the lesions of the vessel wall, intensifying the progression of atherosclerosis. T and B lymphocytes play a key role in the pathogenesis of rheumatological diseases. It is worth mentioning that a specialized population of T lymphocytes, CD4+ CD28- cells, was isolated from inflammatory lesions of the synovium and ruptured plaque [3].

An important factor contributing to the intensification of atherosclerosis progression in rheumatological diseases is the presence of various genetic polymorphisms, such as NFKB1-94ATTG ins/del polymorphism, $H L A-D R B 1^{\star} 0404$ shared epitope allele, and a TNF- $\alpha$ rs1800629 gene polymorphism [3].

From a clinical point of view, the influence of rheumatological disease treatment on the risk of ASCVD is signifi- cant. Ideally, such a therapy would work to reduce the risk of ASCVD. For example, in a study by Ozen et al. [14] was compared the effects of biologic disease-modifying antirheumatic drugs (bDMARD) and conventional synthetic DMARD (cSDMARD) on incident cardiovascular disease in patients with rheumatoid arthritis. Cardiovascular risk reduction with TNF inhibitors ( $\mathrm{HR} 0.81,95 \% \mathrm{Cl}$ : 0.71-0.93) and abatacept ( $\mathrm{HR}=0.50 ; 95 \% \mathrm{Cl}: 0.30-0.83)$ compared to csDMARD was demonstrated. Also, it was found that minimizing the use of glucocorticoids and optimizing the dose of methotrexate may improve cardiovascular outcomes in patients with rheumatoid arthritis [14].

Moreover, a study by Yang et al. [15] assessed the long-term effect of treating patients with systemic lupus erythematosus (SLE) with hydroxychloroquine (HCQ) on the risk of ASCVD. Decreased risk for CAD was found among SLE patients with a high usage of HCQ for at least 318 days ( $\mathrm{HR}=0.31 ; 95 \% \mathrm{Cl}$ : 0.12-0.76). A low risk for CAD was observed in SLE patients with a high cumulative dose of at least $100.267 \mathrm{mg} \mathrm{HCQ}(\mathrm{HR}=0.25$; 95\% Cl: 0.09-0.66) [15].

Thus, patients with rheumatological diseases have an increased risk of ASCVD, which is a result of increased inflammation and autoimmune processes. Treatment of these conditions reduces the risk of ASCVD in this group of patients.

\section{Conclusions}

Atherosclerosis with its background in inflammation and autoimmunity processes is not only a pathology of the cardiovascular system. Lots of autoimmune and rheumatological conditions are currently viewed as risk factors for accelerated atherosclerosis. Thus, cardiovascular check-ups must be included in rheumatology practice. New methods of treatment and new data on some drugs influencing both cardiovascular and rheumatological diseases (like statins) are increasing our mutual recognition of both fields of medicine.

The authors declare no conflict of interest.

\section{References}

1. Ji X, Leng XY, Dong Y, et al. Modifiable risk factors for carotid atherosclerosis: a meta-analysis and systematic review. Ann Transl Med 2019; 7: 632, DOI: 10.21037/atm.2019.10.115.

2. Roth GS, Mensah GA, Johnson CO, et al. Global burden of cardiovascular diseases and risk factors, 1990-2019: update from the GBD 2019 study. J Am Coll Cardiol 2020; 76: 2982-3021, DOI: 10.1016/j.jacc.2020.11.010.

3. Hong J, Maron DJ, Shirai T, Weyand CM. Accelerated atherosclerosis in patients with chronic inflammatory rheumatologic conditions. Int J Clin Rheumtol 2015; 10: 365-381, DOI: 10.2217/ijr.15.33. 
4. Wolf D, Ley K. Immunity and Inflammation in Atherosclerosis. Circ Res 2019; 124: 315-327, DOI: 10.1161/CIRCRESAHA 118.313591.

5. Matsuura E, Atzeni F, Sarzi-Puttini P, et al. Is atherosclerosis an autoimmune disease? BMC Med 2014; 12: 47, DOI: 10.1186/1741-7015-12-47.

6. Cai X, Li J, Cai W, et al. Meta-analysis of type 1 diabetes mellitus and risk of cardiovascular disease. J Diabetes Complications 2021; 35: 107833, DOI: 10.1016/j.jdiacomp.2020.107833.

7. Li H, Tong Q, Guo L, et al. Risk of coronary artery disease in patients with systemic lupus erythematosus: a systematic review and meta-analysis. Am J Med Sci 2018; 356: 451-463, DOI: 10.1016/j.amjms.2018.08.001.

8. Lee TH, Song GG, Choi SJ, et al. Relationship of rheumatoid arthritis and coronary artery disease in the Korean population: a nationwide cross-sectional study. Adv Rheumatol 2019; 59: 40, DOI: 10.1186/s42358-019-0084-6.

9. Man A, Zhu Y, Zhang Y, et al. The risk of cardiovascular disease in systemic sclerosis: a population-based cohort study. Ann Rheum Dis 2013; 72: 1188-1193, DOI: 10.1136/annrheumdis-2012-202007.

10. Prodanovich S, Kirsner RS, Kravetz JD, et al. Association of psoriasis with coronary artery, cerebrovascular, and peripheral vascular diseases and mortality. Arch Dermatol 2009; 145: 700-703, DOI: 10.1001/archdermatol.2009.94.
11. Chen WH, Chen YK, Lin CL, et al. Hashimoto's thyroiditis, risk of coronary heart disease, and L-thyroxine treatment: a nationwide cohort study. J Clin Endocrinol Metab 2015; 100: 109-114, DOI: 10.1210/jc.2014-2990.

12. Palladino R, Marrie RA, Majeed A, Chataway J. Evaluating the risk of macrovascular events and mortality among people with multiple sclerosis in England. JAMA Neurol 2020; 77: 820-828, DOI: 10.1001/jamaneurol.2020.0664.

13. Szymański FM, Mickiewicz A, Dzida G, et al. Leczenie dyslipidemii w Polsce - interdyscyplinarne stanowisko grupy ekspertów wsparte przez Sekcję Farmakoterapii Sercowo-Naczyniowej Polskiego Towarzystwa Kardiologicznego. IV Deklaracja Sopocka. Chor Serca Naczyń 2021; 18: 95-120, DOI: 10.5603/ ChSiN.2021.0011.

14. Ozen G, Pedro S, Michaud K. The risk of cardiovascular events associated with disease-modifying antirheumatic drugs in rheumatoid arthritis. J Rheumatol 2021; 48: 648-655, DOI: 10.3899/jrheum.200265.

15. Yang DH, Leong PY, Sia SK, et al. Long-term hydroxychloroquine therapy and risk of coronary artery disease in patients with systemic lupus erythematosus. J Clin Med 2019; 8: 796, DOI: $10.3390 / \mathrm{jcm} 8060796$. 